\title{
Genetic variations in hypoxia response genes influence hypertrophic cardiomyopathy phenotype
}

\author{
Jaime Alkon'1, Mark K. Friedberg', Cedric Manlhiot' ${ }^{1}$, Ashok Kumar Manickaraj', Caroline Kinnear', Brian W. McCrindle', \\ Leland N. Benson', Linda J. Addonizio², Steven D. Colan ${ }^{3}$ and Seema Mital'
}

BACKGROUND: Risk factors for diastolic dysfunction in hypertrophic cardiomyopathy (HCM) are poorly understood. We investigated the association of variants in hypoxia-response genes with phenotype severity in pediatric HCM.

METHODS: A total of 80 unrelated patients $<21 \mathrm{y}$ and 14 related members from eight families with HCM were genotyped for six variants associated with vascular endothelial growth factor $\mathrm{A}$ (VEGFA) downregulation, or hypoxia-inducible factor A (HIFIA) upregulation. Associations between risk genotypes and leftventricular (LV) hypertrophy, LV dysfunction, and freedom from myectomy were assessed. Tissue expression was measured in myocardial samples from 17 patients with $\mathrm{HCM}$ and 20 patients without HCM.

RESULTS: Age at enrollment was $9 \pm 5$ y (follow-up, $3.1 \pm$ 3.6 y). Risk allele frequency was $67 \%$ VEGFA and $92 \%$ HIF1A. Risk genotypes were associated with younger age at diagnosis $(P<$ 0.001), septal hypertrophy $(P<0.01)$, prolonged E-wave deceleration time (EWDT) $(P<0.0001)$ and isovolumic relaxation time (IVRT) $(P<0.0001)$, and lower freedom from myectomy $(P<$ 0.05). These associations were seen in sporadic and familial HCM independent of the disease-causing mutation. Risk genotypes were associated with higher myocardial HIF1A and transforming growth factor B1 (TGFB1) expression and increased endothelialfibroblast transformation $(P<0.05)$.

CONCLUSION: HIFIA-upregulation and/or VEGFA-downregulation genotypes were associated with more severe septal hypertrophy and diastolic dysfunction and may provide genetic markers to improve risk prediction in HCM.

$\mathrm{H}$ ypertrophic cardiomyopathy (HCM) is the most common cause of sudden death in young athletes (1). Some patients present early with significant hypertrophy and/or sudden death whereas others remain asymptomatic into late adulthood. The source of this variability is poorly understood, with significant gaps in our understanding of the biologic factors involved (2). An important biologic response to the increase in myocardial mass is the induction of angiogenesis to increase myocardial perfusion and oxygen delivery. Frequently, the angiogenic response is inadequate as seen in some HCM cases as a reduction in coronary flow reserve and reduced capillary density and luminal diameter (2-5). Resulting regional perfusion abnormalities probably contribute to cell injury and myocardial fibrosis, an important predictor of adverse outcomes in $\operatorname{HCM}(6,7)$. In an adult series, $71 \%$ of patients with HCM had myocardial fibrosis when assessed by magnetic resonance imaging and serum markers of fibrosis (8). Together, impaired angiogenesis and fibrosis can predispose to arrhythmias and sudden death $(2,3)$. Given the low sensitivity of current imaging methods to identify fibrosis in the early stages, better predictors of impaired angiogenesis and fibrosis are needed.

Hypoxia-inducible factor (HIF1A) is a master regulator of the adaptive response to hypoxia (9). During acute hypoxia, HIF1A stabilizes within the nucleus and binds to the hypoxia-response element of target gene promoters and activates the transcription of vascular endothelial growth factor (VEGF) and metabolic genes to increase angiogenesis and facilitate adaptation to hypoxia (10). Paradoxically, chronic HIF1A stabilization can have a detrimental effect by inducing transforming growth factor B1 (TGFB1) and TGFB1-mediated fibrosis (11-13). TGFB1 promotes the transformation of endothelial lineages into fibroblasts through the reactivation of an embryologic process called endothelial-mesenchymal transition (14). Although a role for the profibrotic cytokine TGFB1 has been reported in a mouse model of HCM (15), its role has not been investigated in HCM in humans. The purpose of our study was to identify the role of hypoxia-signaling pathways in myocardial adaptation in HCM. Specifically, we investigated the association of variations in VEGFA and HIF1A genes with the severity of left-ventricular (LV) hypertrophy, diastolic dysfunction, expression of profibrotic signaling factors, and need for interventions in pediatric HCM.

\section{RESULTS}

\section{SNP Frequencies}

We enrolled 80 index cases: 47 from cohort 1, 33 from cohort 2 . Single-nucleotide polymorphisms (SNP) frequencies were similar in the two cohorts and comparable with those reported in the general population (Table 1) (16-18). The two cohorts were comparable except for a higher frequency of blacks in cohort 1 vs. $2(21 \%$ vs. $0 \%)$. Blacks had a higher frequency than nonblacks of the HIF1A145 T allele ( $78 \%$ vs. $40 \% P=0.001$ ). However, there was no influence of race on the association of genotypes 
with outcomes. All genotypes were in Hardy-Weinberg equilibrium, with no gender-based differences in allele frequencies.

\section{Patient Characteristics}

Table 2 shows the clinical characteristics of the cohort. Fiftyone percent had a positive family history with no difference in age at diagnosis between familial and nonfamilial HCM $(9.3 \pm 5.4$ y vs. $9.1 \pm 6.9$ y, respectively, $P=0.83)$. Diseasecausing mutations were identified in 35 patients; $90 \%$ were sarcomeric. A total of 25 patients (32\%) had a major cardiac event during follow-up, including myectomy or implantable cardioverter defibrillator insertion. Indications for myectomy were LV outflow peak gradient $>100 \mathrm{~mm} \mathrm{Hg}$ with symptoms at rest or during exercise. Indications for implantable cardioverter defibrillator insertion were ventricular tachycardia or ischemic changes with exercise, hypotension at peak exercise, aborted sudden cardiac death, family history of cardiac arrest, or a combination of the above. Baseline echocardiographic data showed elevated interventricular septal (IVS) Z-scores, posterior wall thickness z-scores, LV mass z-scores, and impaired LV relaxation as seen by the prolongation of the isovolumic relaxation time (IVRT) and the E-wave deceleration time (EWDT) (Table 3) $(19,20)$.

Table 1. Allele frequencies

\begin{tabular}{llll}
\hline SNP & rsID & Gene code & $\begin{array}{l}\text { Risk allele } \\
\text { frequency }\end{array}$ \\
\hline VEGFA $(2578 \mathrm{~A} / \mathrm{C})$ & $\mathrm{rs699947}$ & VEGF-1 & $\mathrm{A}(39 \%)$ \\
VEGFA $(1154 \mathrm{~A} / \mathrm{G})$ & $\mathrm{rs} 1570360$ & VEGF-2 & $\mathrm{A}(31 \%)$ \\
VEGFA $(634 \mathrm{C} / \mathrm{G})$ & $\mathrm{rs} 2010963$. & VEGF-3 & $\mathrm{G}(68 \%)$ \\
HIF1A $(145 \mathrm{C} / \mathrm{T})$ & $\mathrm{srs} 10873142$ & HIF1A-1 & $\mathrm{T}(73 \%)$ \\
HIF1A $(1326 \mathrm{C} / \mathrm{T})$ & $\mathrm{rs} 2057482$ & HIF1A-2 & $\mathrm{C}(81 \%)$ \\
HIF1A $(1744 \mathrm{C} / \mathrm{T})$ & $\mathrm{rs} 11549465$ & HIF1A-3 & $\mathrm{C}(92 \%)$ \\
\hline
\end{tabular}

HIF1A, hypoxia-inducible factor 1A; SNP, single-nucleotide polymorphism; VEGF, vascular endothelial growth factor.

Table 2. Baseline clinical characteristics $(n=80)$

\begin{tabular}{lc}
\hline Sex (male) & $52(65 \%)$ \\
Race & \\
White & $43(54 \%)$ \\
Black & $11(13 \%)$ \\
Asian & $11(14 \%)$ \\
Other & $15(19 \%)$ \\
Median age at diagnosis (years) & $9(0.5-19)$ \\
Median age at enrollment (years) & $11(0.9-20)$ \\
Median duration of follow-up (years) & $1.8(0.01-17.5)$ \\
Positive family history & $41(51 \%)$ \\
Genetic testing & $39(49 \%)$ \\
Mutation-positive & $35(90 \%)$ \\
Event rate & \\
Myectomy & $16(20 \%)$ \\
Implantable cardioverter defibrillator & $13(16 \%)$ \\
Cardiac transplant & $3(4 \%)$ \\
Death & $1(1 \%)$ \\
\hline
\end{tabular}

\section{Genotype Associations}

The associations (unadjusted) of risk genotypes with phenotype and the incremental effect of each additional risk genotype are shown in Figures 1-5. Results were similar after adjusting for confounding variables. Results are shown as whisker plots for linear regression analysis of risk genotypes with outcome and as box plots for outcomes within patients carrying 2 vs. 0 or 1 risk alleles for those SNPs that showed significant differences on linear regression analysis.

Age at diagnosis. There was a clustering toward a younger age at diagnosis in patients with VEGFA and HIF1A-risk genotypes (Figure 1a). The box plots show median values with 25 th and 75 th percentiles and overall range in patients with 2 risk alleles vs. 1 or 0 risk alleles for the VEGF-3 and HIF1A-3 genotypes (Figure 1b). A higher number of VEGFA-risk genotypes correlated with a younger age at diagnosis (estimated effect size: -0.84 , confidence interval $-1.53,-0.09$ y per homozygous risk genotype, $P=0.03$ ).

Septal hypertrophy. HIF1A-risk genotypes were associated with higher IVS $\mathrm{z}$-scores with an incremental increase in

Table 3. Baseline echocardiographic data $(n=80)$

\begin{tabular}{|c|c|}
\hline Age at echocardiogram (years) & $11 \pm 5$ \\
\hline Median body surface area $\left(\mathrm{m}^{2}\right)$ & $1.26 \pm 0.54$ \\
\hline Interventricular septum z-score & $6.3 \pm 4$ \\
\hline LV end-diastolic dimension z-score & $-1.5 \pm 2.3$ \\
\hline LV posterior wall thickness z-score & $2.4 \pm 2.3$ \\
\hline LV mass z-score & $5 \pm 7$ \\
\hline LV shortening fraction (\%) & $45 \pm 12$ \\
\hline LV outflow peak gradient $(\mathrm{mm} \mathrm{Hg})$ & $31 \pm 50$ \\
\hline LV ejection fraction (\%) & $67 \pm 10$ \\
\hline \multicolumn{2}{|c|}{ Echocardiographic diastolic parameters $(n=33)$} \\
\hline E wave MV Inflow (cm/s) & $95 \pm 29$ \\
\hline A wave MV Inflow (cm/s) & $60 \pm 26$ \\
\hline E wave deceleration time (ms) & $197 \pm 52$ \\
\hline IVRT (ms) & $83 \pm 26$ \\
\hline Ar PV/A wave & $0.9 \pm 0.4$ \\
\hline LA volume index $\left(\mathrm{cc} / \mathrm{m}^{2}\right)$ & $29 \pm 12$ \\
\hline $\mathrm{Ea} \mathrm{M}(\mathrm{cm} / \mathrm{s})$ & $17 \pm 24$ \\
\hline Aa $M(\mathrm{~cm} / \mathrm{s})$ & $11 \pm 16$ \\
\hline E/Ea M & $10 \pm 4$ \\
\hline $\operatorname{EaS}(\mathrm{cm} / \mathrm{s})$ & $10 \pm 17$ \\
\hline $\operatorname{AaS}(\mathrm{cm} / \mathrm{s})$ & $7 \pm 6$ \\
\hline $\mathrm{E} / \mathrm{EaS}$ & $13 \pm 6$ \\
\hline SAM & $33 \%$ \\
\hline
\end{tabular}

Aa M, A wave by tissue Doppler at the mitral valve annulus; $\mathrm{Aa}$ S, A wave by tissue Doppler at the interventricular septum; Ar PV/A wave, ratio of A wave reversal of the pulmonary venous Doppler and A wave of the mitral valve; $E / E a$, interval between onset of mitral E and annular early diastolic velocity (Ea) by tissue Doppler; Ea M, E wave velocity by tissue Doppler at mitral annulus; Ea S, E wave velocity by tissue Doppler at the interventricular septum; EaT, E wave velocity by tissue Doppler at the tricuspid valve annulus; IVRT, isovolumic relaxation time; LA, left atrium; LV, left-ventricular; MV, mitral valve; SAM, systolic anterior motion of the anterior leaflet of the mitral valve. 
a

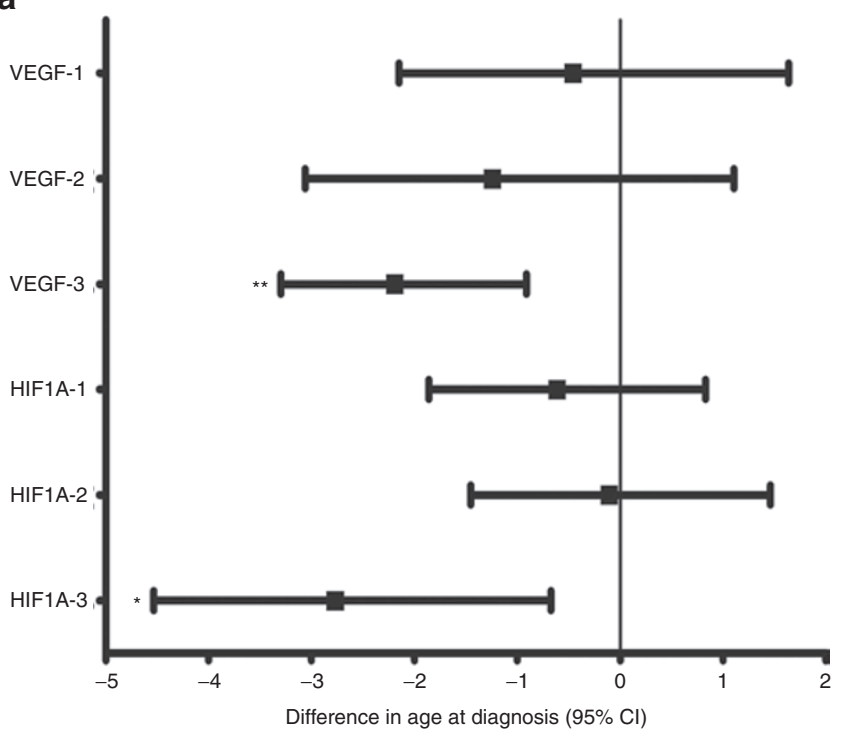

b

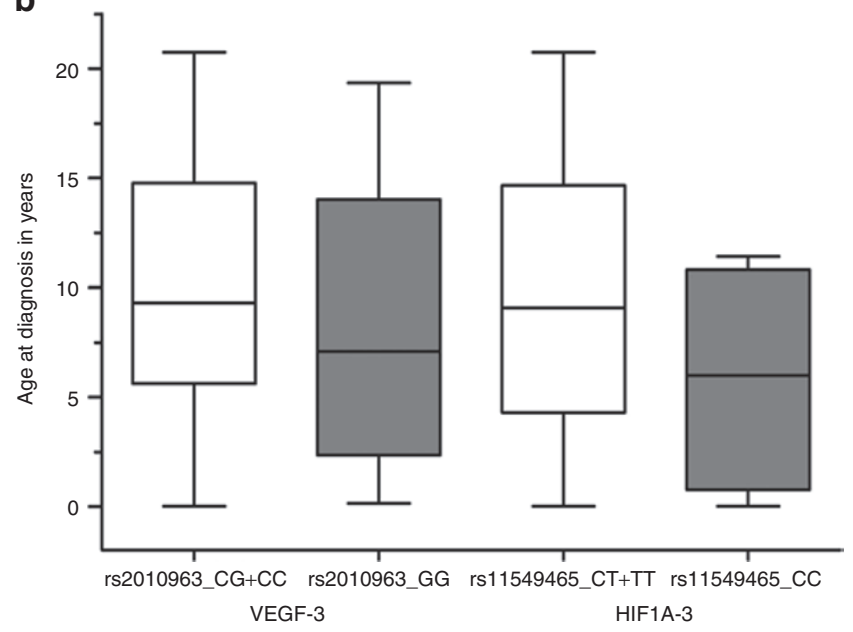

Figure 1. Genotype association with age at diagnosis. (a) Relationship between homozygous risk genotypes and age at diagnosis with earlier age at diagnosis in patients with vascular endothelial growth factor $A$ (VEGFA) and hypoxia-inducible factor 1A (HIF1A) risk genotypes. The $x$ axis shows the mean difference (black squares) between controls and risk genotypes with $95 \%$ confidence intervals. (b) The box plot shows median age at diagnosis, 25th and 75th percentiles, and overall range in patients with $0 / 1$ (white) vs. 2 risk alleles (gray) in VEGF-3 and HIF1A-3 genes. ${ }^{*} P<0.05$, ** $P<0.001$ between risk genotypes and controls. VEGF-1 $=$ VEGFA 2578A/C; VEGF-2 = VEGFA 1154A/G; VEGF-3 = VEGFA 634C/G; HIF1A-1 = HIF1A 145C/T; HIF1A-2 = HIF1A 1326C/T; HIF1A-3 = HIF1A 1744C/T.

IVS z-scores for each additional homozygous risk genotype (estimated effect size: +0.61 , confidence interval $+0.15,+1.11$ $\mathrm{Z}$-score per risk genotype, $P=0.009$ ) (Figure 2a). The box plots show median values with 25 th and 75 th percentiles and overall range in patients with 2 risk alleles vs. 1 or 0 risk alleles for the HIF1A-1 and HIF1A-2 genotypes (Figure 2b).

\section{Diastolic Function}

EWDT. EWDT was significantly prolonged, particularly in those with risk genotypes (Figure 3a). There was an incremental increase in EWDT for each additional VEGFA and HIF1A
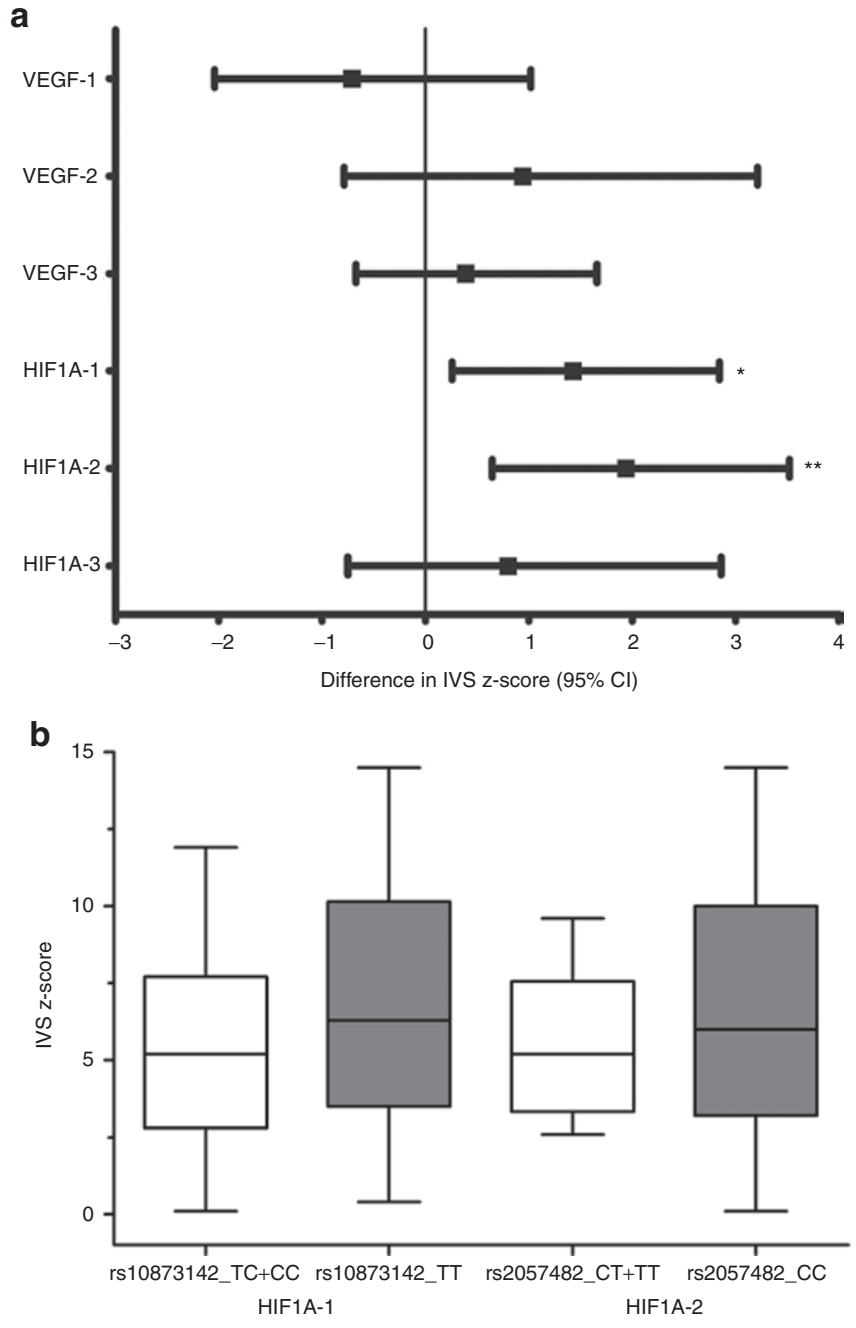

Figure 2. Genotype association with septal hypertrophy. (a) Relationship between homozygous risk genotypes and interventricular septum (IVS) z-scores with higher scores in patients with hypoxia-inducible factor A (HIF1A) risk genotypes. The $x$ axis shows the mean difference (black squares) between controls and risk genotypes with $95 \%$ confidence intervals. (b) The box plot shows median IVS z-scores, 25 th and 75th percentiles, and overall range in patients with $0 / 1$ (white) vs. 2 risk alleles (gray) in HIF1A-1 and HIF1A-2 genes. ${ }^{*} P<0.05,{ }^{* *} P<0.01$ between risk genotypes and controls.

homozygous risk genotype $(P<0.0001)$ (Figure $3 b)$. The box plots show median values with 25 th and 75 th percentiles and overall range in patients with 2 risk alleles vs. 1 or 0 risk alleles for VEGF-1,VEGF-2, VEGF-3, and HIF1A-1 genotypes (Figure 3c).

IVRT. IVRT was prolonged and was associated with a higher number of HIF1A homozygous risk genotypes $(P<0.001)$ (Figure 4a-b). Of note, VEGF-risk genotypes were associated with lower IVRT as compared with $H I F 1 A$-risk genotypes. The box plots show median values with 25 th and 75 th percentiles and overall range in patients with 2 risk alleles vs. 1 or 0 risk alleles for VEGF-1, VEGF-2, HIF1A-1, HIF1A-2, and HIF1A-3 genotypes (Figure $\mathbf{4 c - d}$ ). In addition, VEGFA 2578AA/AC and $1154 A A$ were associated with increased mitral A wave velocity 


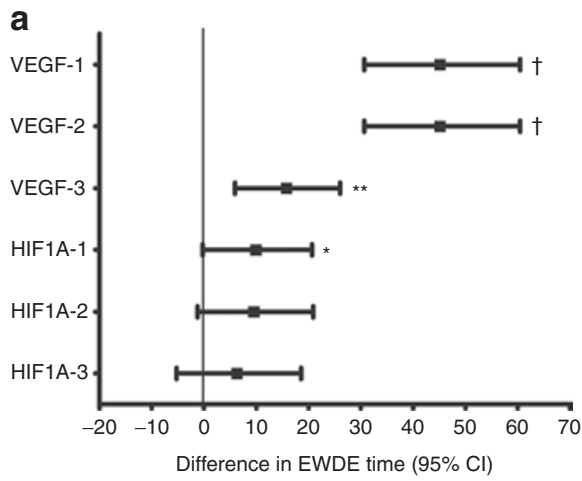

b

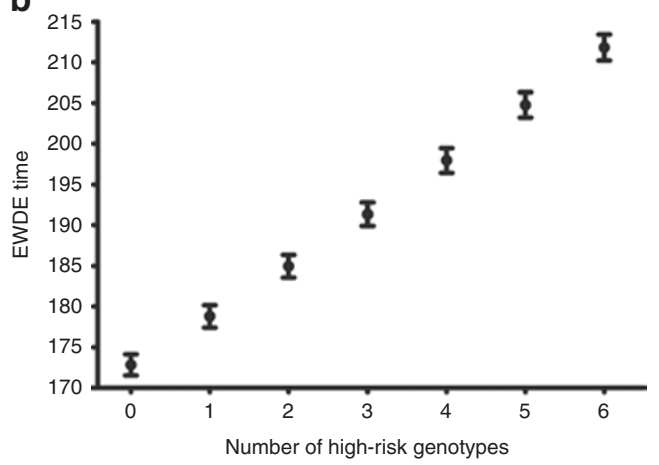

C

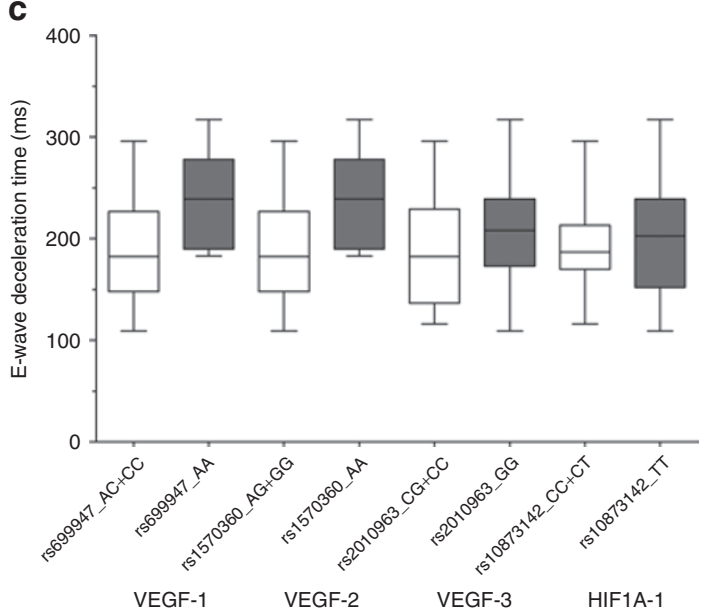

Figure 3. Genotype association with diastolic dysfunction, i.e., E-wave deceleration (EWDE) time. (a) EWDE time was longer in patients with homozygous vascular endothelial growth factor A (VEGFA) and hypoxiainducible factor A (HIF1A) risk genotypes. (b) There was a linear association between the number of risk genotypes and EWDE time $(P=0.001)$. (c) The box plot shows median E-wave deceleration time, 25th and 75th percentiles, and overall range in patients with $0 / 1$ (white) vs. 2 risk alleles (gray) in VEGF-1, VEGF-2, VEGF-3, and HIF1A-1 genes. ${ }^{*} P<0.05,{ }^{* *} P<0.01,{ }^{\dagger} P<0.001$ between risk genotypes and controls.

$(P<0.0001)$. These associations were independent of age, race, familial etiology of HCM, and the presence of sarcomeric mutations. Together, these data demonstrate a significant association of risk genotypes with LV-relaxation abnormalities.

\section{Myectomy}

The following homozygous risk genotypes were associated with a higher hazard ratio (HR) for myectomy (95\% confidence intervals): $\geq 2$ HIF1A-risk genotypes (HR 4.96 (1.08-22.8), $P=$ 0.04), VEGFA1154AA/AG (HR 2.74 (94-7.98), $P=0.06)$, and VEGFA2578AA/AC (HR 4.42 (1.01-10.54), $P=0.05)$. The risk increased incrementally with increasing number of VEGFArisk genotypes (HR: 2.03 (1.06-3.87) per risk genotype, $P=$ 0.03) (Figure 5).

\section{Intrafamilial Replication}

To determine the modifying effect of these genotypes in family members carrying the same sarcomeric mutation, genotypephenotype association analysis was performed in 14 affected members from six families with gene-positive HCM. This included two families (four members) with myosin heavy chain 7 mutations and four families (10 members) with myosin-binding protein $\mathrm{C} 3$ mutations. Affected siblings were matched for age at the time of echocardiography (Table 4). Within each family, patients with $\geq 3$ VEGFA and/or HIF1A homozygous risk genotypes (white bars) had higher IVS z-scores (Figure 6a), longer EWDT, (Figure 6b) and longer IVRT (Figure 6c) as compared with those with $<3$ risk genotypes (black bars), indicating that the modifier effect of the risk genotypes in HCM was independent of the disease causing mutation.

\section{Myocardial Angiogenesis and Fibrosis}

Tissue results are shown in Table 5 with representative images in Figure 7. The non-HCM patients included 12 patients with isolated ventricular septal defects and eight patients with ventricular septal defects with other defects including atrioventricular septal defect or tetralogy of Fallot. The mean age was not different between non-HCM patients $(5.6 \pm 6.2 \mathrm{y}, n=20)$ as compared with HCM patients at surgery $(6.7 \pm 6.1 \mathrm{y}, n=17$, $P=0.6)$. Males were $65 \%$ in both groups. There was stronger VEGF expression $(P=0.001)$; stronger nuclear HIF1A staining in LV in HCM patients as compared with non-HCM patients $(P=0.0003)$; stronger TGFB1 expression $(P=0.009)$; more fibrosis, although this did not reach statistical significance $(P$ $=0.08$ ); and lower capillary density, i.e., von Willebrand factor $(\mathrm{vWF})+$ cells $(P=0.05)$ (Table 6). Seventy-five percent of HCM samples showed vWF + /fibroblast-specific protein + double-positive cells, suggesting the presence of endothelialmesenchymal transition as compared with none in the nonHCM group $(P=0.02)$. Among HCM patients, comparison by genotype showed the association of VEGFA-risk genotypes with weaker myocardial VEGF expression (Figure 7a-d) and more endothelial-mesenchymal transition as compared with those without VEGFA risk alleles (data not shown). HI1F1Arisk genotypes were associated with stronger nuclear HIF1A expression (Figure 7e-h), TGFB1 expression (Figure 7i-l), and more endothelial-mesenchymal transition as compared with patients lacking HIF1A-risk alleles (Figure 7m-p).

\section{DISCUSSION}

Our study shows that genetic variations in angiogenic and hypoxia-response genes, VEGFA and HIF1A, were associated with younger age at diagnosis, greater septal hypertrophy, diastolic dysfunction, lower freedom from myectomy, and increased 

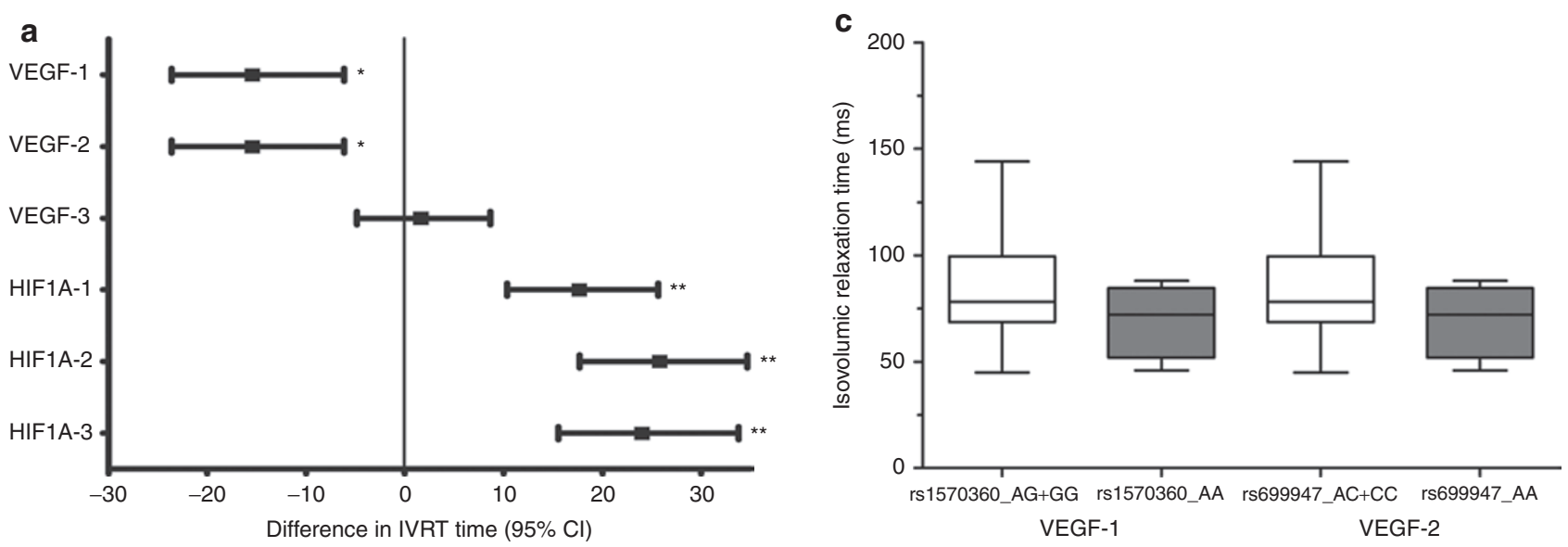

b

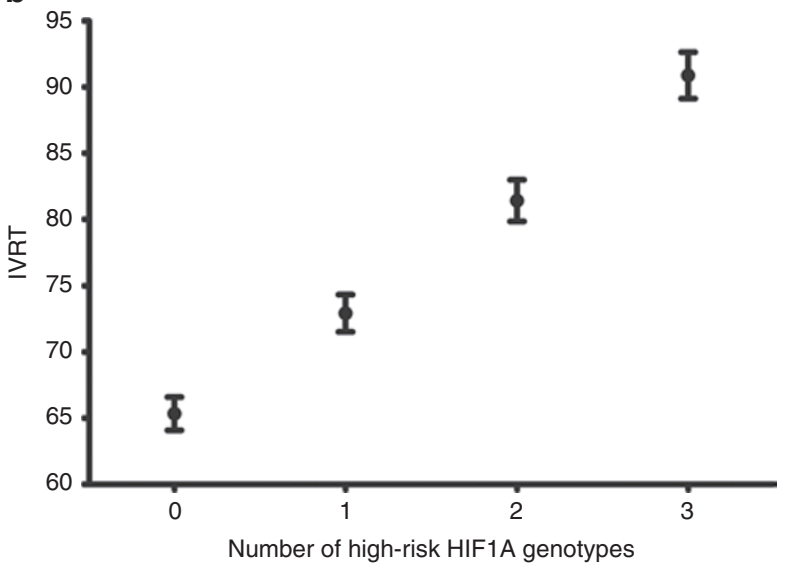

d

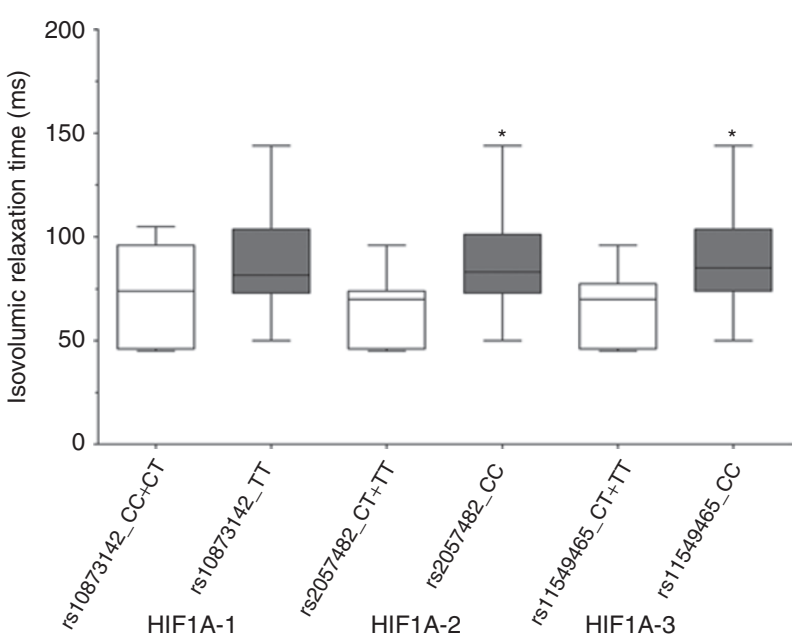

Figure 4. Genotype association with diastolic dysfunction, i.e., isovolumic relaxation time (IVRT). (a) IVRT was longer in patients with homozygous hypoxia-inducible factor (HIF1A) risk genotypes. (b) There was a linear association between the number of HIF1A risk genotypes and IVRT $(P<0.001)$. The box plots show median IVRT, 25th and 75th percentiles, and overall range in patients with $0 / 1$ (white) vs. 2 risk alleles (gray) in (c) vascular endothelial growth factor (VEGF)-1 and VEGF-2 genes, and (d) HIF1A-1, HIF1A-2, and HIF1A-3 genes. ${ }^{*} P<0.05,{ }^{* *} P<0.01$ between risk genotypes and controls.

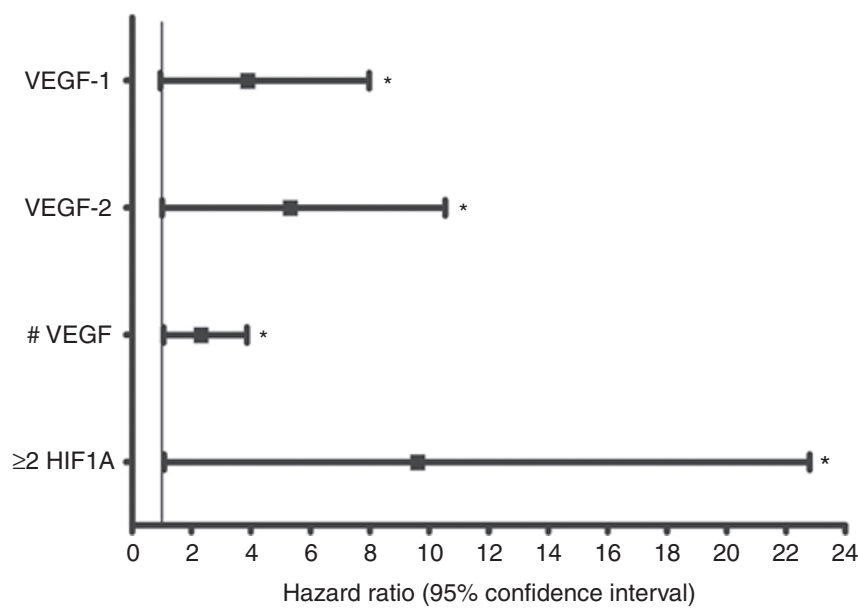

Figure 5. Genotype association with freedom from myectomy. Hazard ratio (HR) for myectomy was higher in patients with vascular endothelial growth factor (VEGFA)-1 and VEGFA-2 risk genotypes, and in patients with $\geq 2$ HIF1A risk genotypes, with an incremental increase in risk with each additional $V E G F A$ risk genotype, i.e., \#VEGF. ${ }^{*} P<0.05$ between risk genotypes vs. controls. Vertical line indicates HR of 1. HIF1A, hypoxia-inducible factor $1 \mathrm{~A}$. myocardial HIF1A expression, increased TGFB1 expression, and increased endothelial-mesenchymal transition. These associations were observed in both unrelated and familial HCM cases and provide a potential mechanism for reduced angiogenesis and fibrosis in HCM, and also provide genetic markers that may prove useful in early risk stratification in HCM.

Despite significant advances in our ability to identify the genetic causes of HCM, our ability to predict outcomes remains poor, largely due to the tremendous phenotypic variability even among patients carrying the same mutations (21). Current risk prediction models rely on clinical and echocardiographic factors that have limited sensitivity, particularly, in the pediatric age range. We studied genetic markers in highly conserved hypoxia and angiogenic genes to help in early identification of patients at risk for disease progression (22-24). VEGF-downregulation and HIF1A-upregulation variants were associated with impaired angiogenesis, increased fibrosis, and downstream ventricular relaxation abnormalities. There was a linear correlation between number of risk genotypes and worsening phenotype, which was independent of the underlying 
Table 4. Intrafamilial comparisons

\begin{tabular}{lllccc}
\hline & $\begin{array}{l}\text { Sarcomeric } \\
\text { mutation }\end{array}$ & Relationship & $\begin{array}{c}\text { Age } \\
\text { (years) }\end{array}$ & $\begin{array}{c}\text { No. of } \\
\text { VEGFA risk } \\
\text { genotypes }\end{array}$ & $\begin{array}{c}\text { No. of } \\
\text { HIF1A risk } \\
\text { genotypes }\end{array}$ \\
\hline Family 1 & MYH7 & Proband & 17 & 0 & 3 \\
Family 2 & MYH7 & Brother & 16.6 & 1 & 3 \\
& & Proband & 14.4 & 2 & 2 \\
Family 3 & MYBPC3 & Brother & 13.9 & 2 & 2 \\
& & Proband & 14.2 & 1 & 3 \\
Family 4 & MYBPC3 & Proband & 10.6 & 1 & 3 \\
& & Brother & 12.4 & 1 & 3 \\
& & Father & 48.4 & 1 & 3 \\
Family 5 & MYBPC3 & Brother & 7.8 & 0 & 3 \\
& & Proband & 17.8 & 3 & 3 \\
Family 6 & MYBPC3 & Proband & 9.5 & 3 & 3 \\
& & Brother & 10.1 & 3 & 3
\end{tabular}

HIF1A, hypoxia-inducible factor A; MYH7, myosin heavy chain 7; MYBPC3, myosin binding protein C3; VEGFA, vascular endothelial growth factor $\mathrm{A}$.

disease-causing mutation. These associations were reproducible in affected family members carrying the same mutations. To assess whether the genotypes were influencing protein expression in the target organ, we measured VEGF and HIF1A expression in the LV myocardium of patients with HCM undergoing myectomy and found that protein expression correlated with genotype, i.e., lower myocardial VEGF expression correlated with loss of function VEGFA genotypes and higher HIF1A expression correlated with functioning HIF1A genotypes. Validation in the target organ or tissue is important to attribute phenotypic variation to genotype differences.

Histologic abnormalities were seen in the context of diastolic dysfunction. Analysis of diastolic dysfunction revealed strong association of risk genotypes with impaired LV relaxation as measured by increased mitral A wave, prolonged mitral EWDT, and prolonged IVRT (25-27). Of note, VEGFA-risk genotypes were associated with lower IVRT as compared with HIF1Arisk genotypes, which may reflect a trend toward a restrictive physiology. However, there was no other evidence of restrictive physiology, suggesting that this young patient cohort was in relatively early stages of diastolic dysfunction. Of note, there was also an independent association with septal hypertrophy, suggesting that patients with risk genotypes have thicker and stiffer LV outflow tracts, accounting for the lower freedom from myectomy in the risk groups. We did not find significant associations with the E/Ea ratio, which has been used as an indicator of filling pressures in adult HCM (28). However, regional heterogeneity in HCM may have precluded reliable analysis by this method $(29,30)$. Of note, the association with diastolic dysfunction was independent of age, race, etiology of HCM, and the severity of septal hypertrophy. Again, these associations were replicated in intrafamilial comparisons that also revealed an effect of genotype on diastolic function.
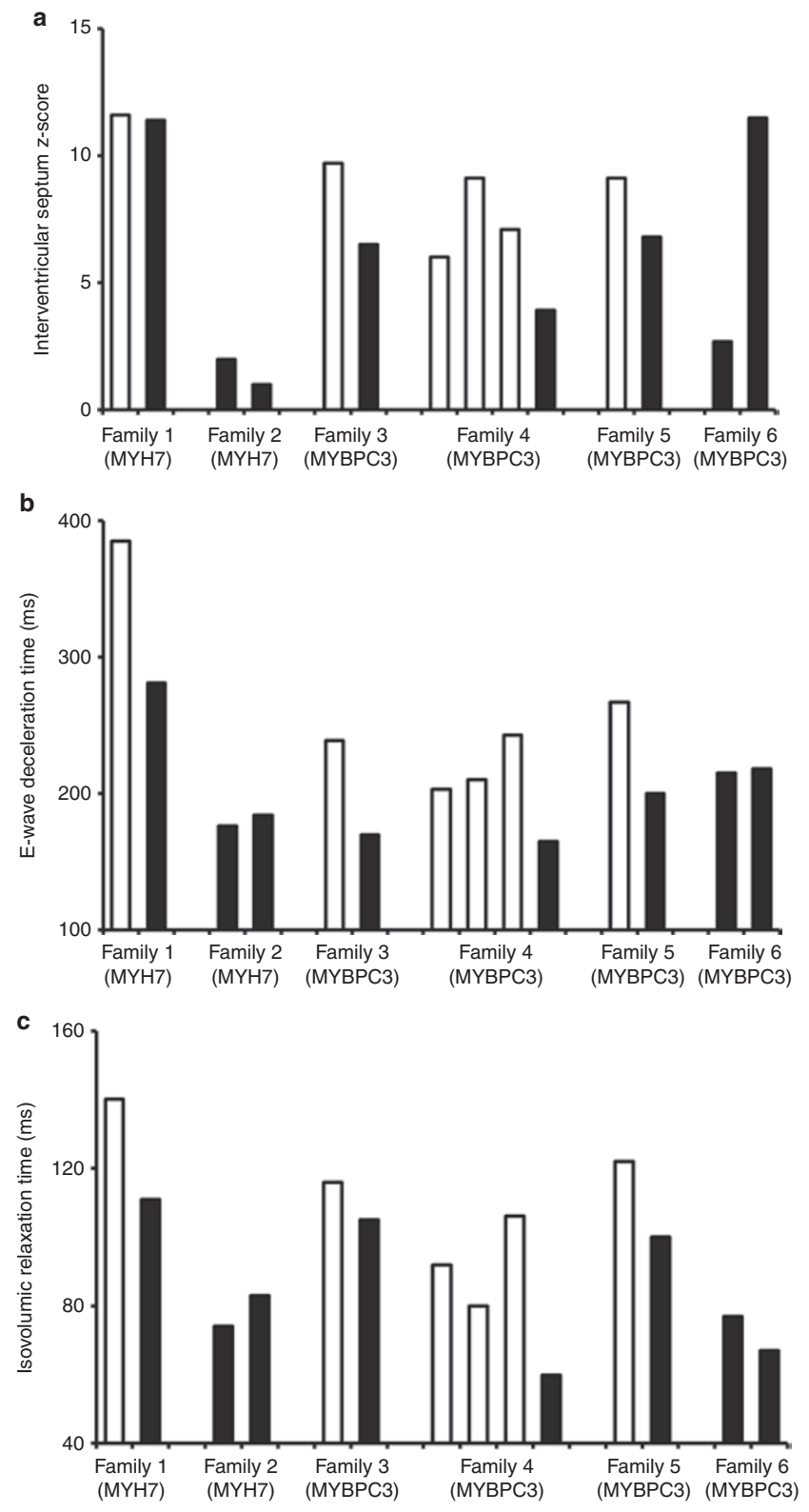

Figure 6. Intrafamilial comparison of genotype-phenotype associations. Echocardiographic comparison in 14 affected members from six gene-positive families, two with MYH7 mutations and four with MYBPC3 mutations. Black bars represent $\leq 3$ hypoxia-inducible factor A (HIF1A) and/or vascular endothelial growth factor $\mathrm{A}$ (VEGFA) risk genotypes; white bars represent $>3$ HIF1A and/or VEGFA risk genotypes. Compared with subjects with $\leq 3$ VEGFA or HIF1A homozygous risk genotypes (black bars), those with $>3$ risk genotypes (white bars) had (a) higher interventricular septum z-scores; (b) longer E wave deceleration time; and (c) longer isovolumic relaxation time. MYH7, myosin heavy chain 7; MYBPC3, myosin binding protein $\mathrm{C} 3$.

To determine if effect on diastolic function was mediated by reduced angiogenesis and increased fibrosis, we assessed myocardial angiogenic and profibrotic signaling across genotypes. Overall, patients with HCM had lower capillary density despite higher myocardial expression of HIF1A and VEGF but higher TGFB1 expression and more evidence of endothelialmesenchymal transition. Endothelial-mesenchymal transition is an embryologic process involved in valuvuloseptal 
Table 5. Myocardial expression profile

\begin{tabular}{lccc}
\hline & HCM & Non-HCM & $P$ \\
\hline$n$ & 17 & 20 & \\
Age at surgery (years) & $7.6 \pm 6.7$ & $10.2 \pm 5.4$ & 0.34 \\
$\begin{array}{l}\text { Cells with nuclear HIF1A } \\
\text { localization (\%) }\end{array}$ & $66 \pm 19$ & $19 \pm 32$ & 0.0003 \\
VEGF expression (relative units) & $2.4 \pm 0.6$ & $1.5 \pm 0.5$ & 0.001 \\
TGFB1 expression (relative units) & $2.7 \pm 1.1$ & $1.6 \pm 0.5$ & 0.009 \\
Capillary density & $13 \pm 6$ & $19 \pm 6$ & 0.05 \\
(vWF + cells/total cells) & & & \\
Myocardial fibrosis area ( $\mu$ m $^{2}$ ) & $26 \pm 19$ & $13 \pm 3$ & 0.08 \\
Patients with vWF+/FSP1+cells (\%) & 75 & 0 & 0.02 \\
\hline
\end{tabular}

HCM, hypertrophic cardiomyopathy; HIF1A, hypoxia-inducible factor A; FSP, fibroblastspecific protein; TGFB1, transforming growth factor B1; VWF, von Willebrand factor; VEGF, vascular endothelial growth factor. morphogenesis in the developing heart. The finding of endothelial-mesenchymal transition in HCM suggests a recapitulation of the embryonic phenotype, probably in response to tissue hypoxia and pressure overload, similar to findings in mouse hearts subjected to pressure overload $(14,31)$. This biologic response to pressure overload and hypoxia may account for the depletion of endothelial lineages and increase in fibroblast transformation. Although we were unable to quantify the proportion of fibroblasts derived from endothelial cells, reports suggest that $\sim 30 \%$ of fibroblasts can be of endothelial origin in disease (32).

Genotype association analysis revealed that these tissue-level changes were more marked in patients with risk genotypes. Patients with more VEGFA downregulation or HIF1Aupregulation genotypes had lower VEGFA, higher myocardial
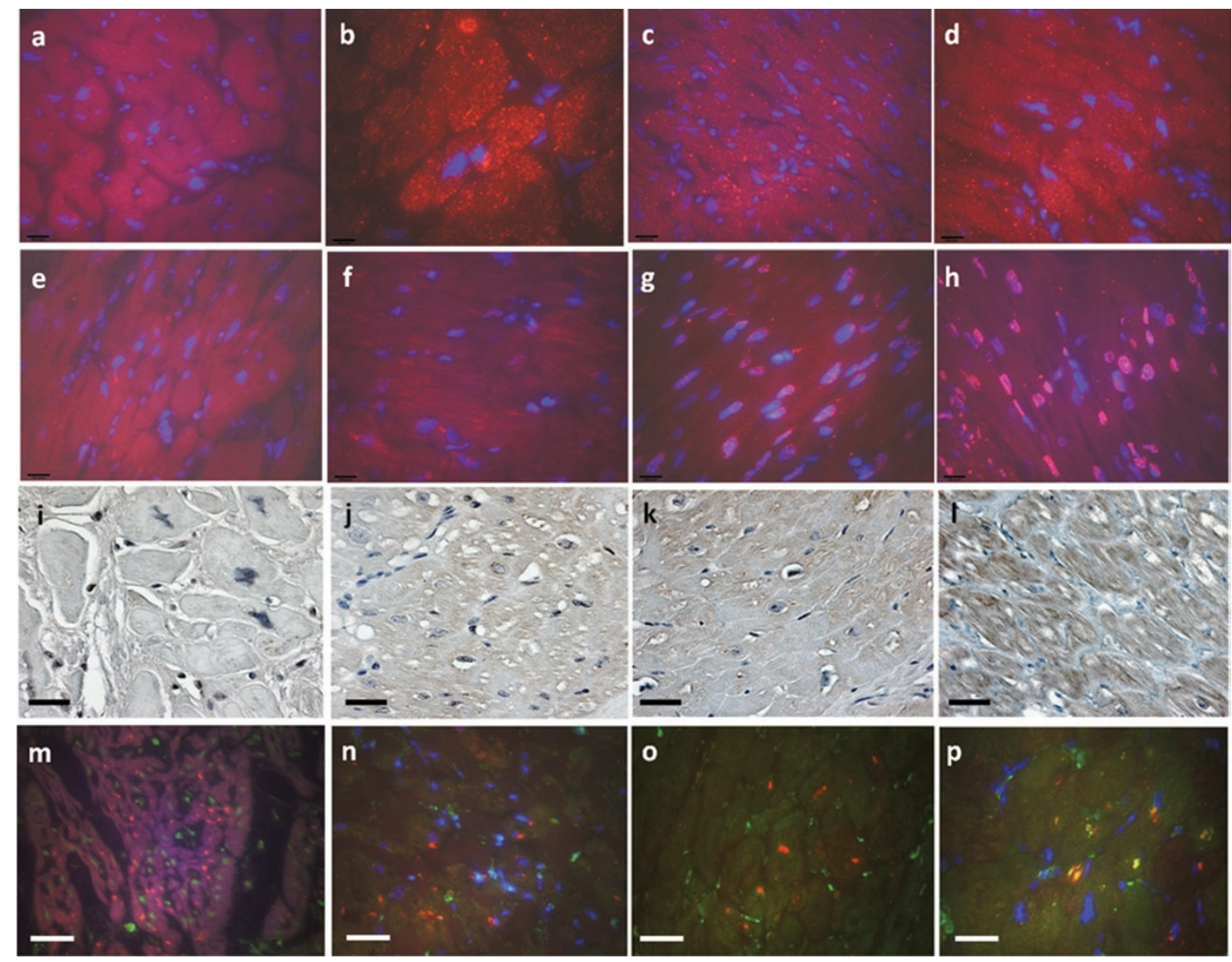

Figure 7. Genotype association with left ventricular myocardial protein expression. Representative immunostaining images of myocardial sections from 17 patients with hypertrophic cardiomyopathy (HCM) and 20 non-HCM patients. Blue = nuclear 4',6-diamidino-2-phenylindole staining. Top row: myocardial vascular endothelial growth factor (VEGF) expression (red) was weaker in non-HCM (a) as compared with HCM samples. Within HCM, VEGF expression was stronger in patients with 0 (b), and weaker in patients with 1 (c) or 2 (d) VEGFA loss-of-function alleles (50× original magnification). Second row: nuclear hypoxia-inducible factor A (HIF1A) expression (red) was weaker in non-HCM (e) as compared with HCM samples. Within HCM samples, nuclear HIF1A expression was weaker in those with 0 functioning HIF1A alleles (f) and stronger in patients with 1 (g) or 2 (h) functioning HIF1A alleles (50x original magnification). Third row: TGFB1 expression (brown) was weaker in non-HCM (i) as compared with HCM samples. Within HCM samples, TGFB1 expression was weaker in those with 0 (j) functioning HIF1A alleles and stronger in patients with 1 (k) or 2 (I) functioning HIF1A alleles $(400 \times$ original magnification; scale bar $35 \mu \mathrm{m})$. Bottom row: capillary density (ratio of vWF + cells to 4',6-diamidino-2-phenylindole+ nuclei) was higher whereas the number of VWF + (red)/fibroblast-specific protein + (green) double-positive cells seen in yellow was lower in non-HCM (m) compared with HCM samples, indicating increased endothelial-mesenchymal transition in HCM. Within HCM, patients with 0 (n) or 1 (o) functioning HIF1A alleles had less endothelial-mesenchymal transition as compared with those with 2 (p) functioning HIF1A alleles (600× original magnification; scale bar $20 \mu \mathrm{m}$ ). vWF, von Willebrand factor. 
HIF1A and TGFB1 expression, and more endothelial-mesenchymal transition. This finding implicates HIF1A and VEGF signaling as potentially important contributors to myocardial fibrosis in HCM and highlights how genetic variation in this pathway can influence myocardial adaptation in HCM. Our ability to identify genetic markers that predispose to a profibrotic phenotype provides us with potentially valuable tools for risk stratification.

\section{Limitations}

Genetic test results were positive in only half the patients, reflecting the lower yield of genetic testing in the early years of this study. However, associations were reproducible in mutation-positive and mutation-negative patients, both unrelated and familial, suggesting that these modifier genes can influence phenotype independent of the disease etiology. The ability to replicate the associations across two geographic cohorts suggests that the findings may be generalizable across populations. The association of genotype with cardiac expression of the encoded proteins, angiogenesis, and fibrosis provides supportive evidence for the observed phenotypic associations. Although we did not perform routine magnetic resonance imaging, which can noninvasively detect myocardial fibrosis in late stages, its ability to detect early fibrosis is questionable as shown in a recent study of adult $\operatorname{HCM}(8,33)$.

In summary, our study shows that diastolic dysfunction in HCM may be regulated by genetic factors that influence myocardial HIF1A, VEGFA, and TGFB1 expression, angiogenesis, and fibrosis. Prospective validation is required to assess the application of these new genetic markers toward risk stratification in HCM. Further studies will identify whether these at-risk patients may benefit from closer surveillance and antifibrotic therapies that inhibit TGFB1.

\section{METHODS}

In a prospective cohort study, unrelated $\mathrm{HCM}$ cases $<21$ y were enrolled at two locations: cohort 1, New York (2005-2007) and cohort 2, Toronto (2007-2010). Patients with significant valvular insufficiency, hypertension, congenital heart disease, and metabolic disorders were excluded. For intrafamilial comparisons, members from families with known mutations were enrolled and analyzed for replication of genotype associations. The study protocol conformed to the ethical guidelines of the 1975 Declaration of Helsinki; the study was approved by the institutional review boards of the Hospital for Sick Children and Morgan Stanley Children's Hospital of New York-Presbyterian, and informed consent was obtained from all subjects or parents/legal guardians. The authors of this article have certified that they comply with the Principles of Ethical Publishing in the International Journal of Cardiology (34).

\section{Clinical and Echocardiographic Data}

Clinical data were obtained from questionnaires and medical records, including demographics, positive family history, presence and type of HCM-causing mutations, and frequency of major cardiac events-myectomy, implantable cardioverter defibrillator insertion, heart transplantation, and death. All patients underwent two-dimensional echocardiography using IE-33 (Philips Ultrasound, Bothell, WA) or Vivid 7 (GE, Horten, Norway) systems. Measurements were made offline by a single investigator (J.A.) who was blinded to subject genotype using commercially available software (Syngo Dynamics, Siemens, Malvern, PA). Echocardiographic data were obtained at last follow-up (before myectomy or transplantation).

\section{Echocardiographic Assessment}

This included two-dimensional measurements of LV dimensions in para-sternal long or short axis planes, LV ejection fraction, maximal IVS and LV posterior wall thickness, peak LV outflow tract gradient at rest, left atrial volume (calculated by biplane Simpson's method of discs from the four- and two-chamber views) indexed to body surface area and LV mass (area-length method). Measurements were made offline from digitally stored images using the average from three consecutive cardiac cycles. LV mass, IVS, and posterior wall thickness z-scores were calculated as previously described $(19,20,35)$. Detailed echocardiographic evaluation of diastolic function using tissue Doppler imaging was performed in a subset of 33 patients. This included mitral and tricuspid valve inflow patterns using pulsed Doppler to determine peak E-wave (early diastolic filling) and A-wave (late diastolic filling) velocities, mitral EWDT, and LV IVRT. Pulmonary venous Doppler assessment, including A-wave reversal duration, was obtained by pulsed wave Doppler from the apical four-chamber view $(36,37)$. Impaired $\mathrm{LV}$ relaxation was defined by the presence of reduced transmitral $\mathrm{E}$ wave velocity, increased A wave velocity, reduced E/A ratio, prolonged EWDT, and prolonged IVRT. LV restriction was defined by increased transmitral E wave velocity, decreased A wave velocity, increased E/A ratio, shortened EWDT, and increased duration of A wave reversal of the pulmonary venous Doppler to the A wave of the mitral valve.

\section{Genotyping}

Blood or saliva was obtained for DNA extraction. Genotyping was performed for SNPs chosen based on previous association studies, functional effects, allele frequency, and prevalence (16-18): VEGFA 2578A/C, VEGFA $1154 \mathrm{~A} / \mathrm{G}$, both SNPs in the VEGF promoter, VEGFA 634C/G in the $5^{\prime}$ untranslated region, HIF1A 145C/T, HIF1A $1326 \mathrm{C} / \mathrm{T}$, and HIF1A $1744 \mathrm{C} / \mathrm{T}$ in the $3^{\prime}$ untranslated region (Table 6). Functioning alleles (major alleles) in HIF1A and loss-of-function alleles (minor alleles) in VEGFA were considered risk alleles. These alleles have shown previous associations with coronary artery disease and chronic heart failure (16,17,38-41). SNP assays were performed using Applied Biosystems Taqman SNP genotyping technology (Assays-by-Design, Burlington, Canada).

\section{Myocardial Studies}

Myocardial samples were obtained from 17 patients with HCM and 20 patients with ventricular septal defects undergoing cardiac surgery. Immunostaining was performed on paraffin-embedded $5 \mu \mathrm{m}$ sections to measure fibrosis area, HIF1, VEGF, TGFB1, vWF, and fibroblastspecific protein expression. Fibrosis area was measured on Masson's trichrome stain (1,000× magnification) using GSA Image Analyzer (GSA Bansemer \& Scheel GbR, Rostock, Germany). Cells with nuclear HIF1A + accumulation were expressed as a percentage of total cells.

Table 6. Primer sequences for VEGF and HIF1A genotype assays VEGFA 2578A/C (rs699947) (VEGF-1)

GCCAGCTGTAGGCCAGACCCTGGCA[A/C]GATCTGGGTGGATAATCAGACTGAC VEGFA 1154A/G (rs1570360) (VEGF-2)

AGCCCGGGCCCGAGCCGCGTGTGGA[A/G]GGGCTGAGGCTCGCCTGTCCCCGCC VEGFA 634C/G (rs2010963) (VEGF-3) CGCGCGGGCGTGCGAGCAGCGAAAG[C/G]GACAGGGGCAAAGTGAGTGACCTGC HIF1A 145C/T (rs10873142) (HIF1A-1) TTAAAACCCTAAATGTGACACAGTA[C/T]GCATGAGTGATCATGCATCTCAAGA HIF1A 1326C/T (rs2057482) (HIF1A-2) CCTTTTTTTGGACACTGGTGGCTCA[C/T]TACCTAAAGCAGTCTATTTATATTT HIF1A 1744C/T (rs11549465) (HIF1A-3) GTTACGTTCCTTCGATCAGTTGTCA[C/T]CATTAGAAAGCAGTTCCGCAAGCCC

HIFIA, hypoxia-inducible factor 1; VEGF, vascular endothelial growth factor. From NCBI dbSNP public database (http://www.ncbi.nlm.nih.gov/SNP/). 
Capillary density was expressed as a ratio of $\mathrm{vWF}+$ to total cells. Colocalization of vWF and fibroblast-specific protein, a fibroblast marker (Abcam, Cambridge, MA), was used as a marker of endothelial-mesenchymal transition. The intensity of $\mathrm{vWF} /$ fibroblast-specific protein, VEGF, and TGFB1 expression was semiquantitatively graded as $1=$ none, 2 = mild-moderate, and $3=$ severe. The investigator was blinded to patient genotype.

\section{Statistical Analysis}

Linear and logistic univariable regression models with maximum likelihood algorithms for parameter estimation were used to test for associations between risk genotypes (homozygous and heterozygous) and outcomes. The differences in outcomes between genotype groups were reported as estimated effect sizes with $95 \%$ confidence intervals for linear regression and HRs with 95\% confidence intervals for logistic regression models. Mann-Whitney $\mathrm{U}$ test was used to compare differences between genotype groups. Models with time-dependent outcomes were adjusted for duration of follow-up, as well as for interaction with family history, sarcomeric mutations, age at diagnosis, and race. Tissue results were compared between HCM and non-HCM samples and in HCM patients with and without risk alleles using Student's $t$ test. Cohorts 1 (New York) and 2 (Toronto) were analyzed separately and jointly with similar results; hence, only data from joint analysis is presented, an approach that has been successfully used in other studies on genomics to improve efficiency and power $(42,43)$. To reduce the risk of false-positive associations with multiple testing, we applied a false discovery rate of $20 \%$ and reported associations showing a cumulative effect of multiple SNPs. $P<0.05$ was used to define statistically significant differences. All statistical analyses were performed using SAS v.9.1 (The SAS Institute, Cary, NC).

\section{STATEMENT OF FINANCIAL SUPPORT}

This work was supported by the Children's Cardiomyopathy Foundation, the SickKids Labatt Family Heart Centre Innovations Fund, and the Roma and Marvin Auerback Endowment Funds.

Disclosure: The authors declared no conflict of interest.

\section{ACKNOWLEDGMENTS}

We acknowledge the Labatt Family Heart Centre Biobank for access to DNA and tissue samples, and The Centre for Applied Genomics (www.tcag.ca/) for genotyping of candidate SNPs.

\section{REFERENCES}

1. Maron BJ, Pelliccia A, Spirito P. Cardiac disease in young trained athletes. Insights into methods for distinguishing athlete's heart from structural heart disease, with particular emphasis on hypertrophic cardiomyopathy. Circulation 1995;91:1596-601.

2. Schwartzkopff B, Mundhenke M, Strauer BE. Alterations of the architecture of subendocardial arterioles in patients with hypertrophic cardiomyopathy and impaired coronary vasodilator reserve: a possible cause for myocardial ischemia. J Am Coll Cardiol 1998;31:1089-96.

3. Kofflard MJ, Michels M, Krams R, et al. Coronary flow reserve in hypertrophic cardiomyopathy: relation with microvascular dysfunction and pathophysiological characteristics. Neth Heart J 2007;15:209-15.

4. Johansson B, Mörner S, Waldenström A, Stål P. Myocardial capillary supply is limited in hypertrophic cardiomyopathy: a morphological analysis. Int J Cardiol 2008;126:252-7.

5. Takemura G, Takatsu Y, Fujiwara H. Luminal narrowing of coronary capillaries in human hypertrophic hearts: an ultrastructural morphometrical study using endomyocardial biopsy specimens. Heart 1998;79: 78-85.

6. Bruder O, Wagner A, Jensen CJ, et al. Myocardial scar visualized by cardiovascular magnetic resonance imaging predicts major adverse events in patients with hypertrophic cardiomyopathy. J Am Coll Cardiol 2010;56:875-87.

7. O'Hanlon R, Grasso A, Roughton M, et al. Prognostic significance of myocardial fibrosis in hypertrophic cardiomyopathy. J Am Coll Cardiol 2010;56:867-74.
8. Ho CY, López B, Coelho-Filho OR, et al. Myocardial fibrosis as an early manifestation of hypertrophic cardiomyopathy. N Engl J Med 2010;363:552-63.

9. Wang GL, Jiang BH, Rue EA, Semenza GL. Hypoxia-inducible factor 1 is a basic-helix-loop-helix-PAS heterodimer regulated by cellular $\mathrm{O}_{2}$ tension. Proc Natl Acad Sci USA 1995;92:5510-4.

10. Rey S, Semenza GL. Hypoxia-inducible factor-1-dependent mechanisms of vascularization and vascular remodelling. Cardiovasc Res 2010;86:23642.

11. Halberg N, Khan T, Trujillo ME, et al. Hypoxia-inducible factor 1alpha induces fibrosis and insulin resistance in white adipose tissue. Mol Cell Biol 2009;29:4467-83.

12. Kimura K, Iwano M, Higgins DF, et al. Stable expression of HIF-1alpha in tubular epithelial cells promotes interstitial fibrosis. Am J Physiol Renal Physiol 2008;295:F1023-9.

13. Moon JO, Welch TP, Gonzalez FJ, Copple BL. Reduced liver fibrosis in hypoxia-inducible factor-1alpha-deficient mice. Am J Physiol Gastrointest Liver Physiol 2009;296:G582-92.

14. Zeisberg EM, Tarnavski O, Zeisberg M, et al. Endothelial-to-mesenchymal transition contributes to cardiac fibrosis. Nat Med 2007;13:952-61.

15. Teekakirikul P, Eminaga S, Toka O, et al. Cardiac fibrosis in mice with hypertrophic cardiomyopathy is mediated by non-myocyte proliferation and requires Tgf- $\beta$. J Clin Invest 2010;120:3520-9.

16. Zheng ZL, Hwang YH, Kim SK, et al. Genetic polymorphisms of hypoxiainducible factor-1 alpha and cardiovascular disease in hemodialysis patients. Nephron Clin Pract 2009;113:c104-11.

17. van der Meer P, De Boer RA, White HL, et al. The VEGF +405 CC promoter polymorphism is associated with an impaired prognosis in patients with chronic heart failure: a MERIT-HF substudy. J Card Fail 2005;11:279-84.

18. Hsieh YY, Wang JP, Lin CS. Four novel single nucleotide polymorphisms within the promoter region of $\mathrm{p} 53$ gene and their associations with uterine leiomyoma. Mol Reprod Dev 2007;74:815-20.

19. Foster BJ, Mackie AS, Mitsnefes M, Ali H, Mamber S, Colan SD. A novel method of expressing left ventricular mass relative to body size in children. Circulation 2008;117:2769-75.

20. Lang RM, Bierig M, Devereux RB, et al. Recommendations for chamber quantification: a report from the American Society of Echocardiography's Guidelines and Standards Committee and the Chamber Quantification Writing Group, developed in conjunction with the European Association of Echocardiography, a branch of the European Society of Cardiology. J Am Soc Echocardiogr 2005;18:1440-63.

21. Ackerman MJ. Genetic testing for risk stratification in hypertrophic cardiomyopathy and long QT syndrome: fact or fiction? Curr Opin Cardiol 2005;20:175-81.

22. Friehs I, Barillas R, Vasilyev NV, Roy N, McGowan FX, del Nido PJ. Vascular endothelial growth factor prevents apoptosis and preserves contractile function in hypertrophied infant heart. Circulation 2006;114:Suppl 1:I290-5.

23. Friehs I, Margossian RE, Moran AM, Cao-Danh H, Moses MA, del Nido PJ. Vascular endothelial growth factor delays onset of failure in pressureoverload hypertrophy through matrix metalloproteinase activation and angiogenesis. Basic Res Cardiol 2006;101:204-13.

24. Sano M, Minamino T, Toko H, et al. p53-induced inhibition of Hif- 1 causes cardiac dysfunction during pressure overload. Nature 2007;446:444-8.

25. Maron BJ, Spirito P, Green KJ, Wesley YE, Bonow RO, Arce J. Noninvasive assessment of left ventricular diastolic function by pulsed Doppler echocardiography in patients with hypertrophic cardiomyopathy. J Am Coll Cardiol 1987;10:733-42.

26. Gidding SS, Snider AR, Rocchini AP, Peters J, Farnsworth R. Left ventricular diastolic filling in children with hypertrophic cardiomyopathy: assessment with pulsed Doppler echocardiography. J Am Coll Cardiol 1986;8:310-6.

27. Matsumura Y, Elliott PM, Virdee MS, Sorajja P, Doi Y, McKenna WJ. Left ventricular diastolic function assessed using Doppler tissue imaging in patients with hypertrophic cardiomyopathy: relation to symptoms and exercise capacity. Heart 2002;87:247-51. 


\section{Articles | Alkon et al.}

28. McMahon CJ, Nagueh SF, Pignatelli RH, et al. Characterization of left ventricular diastolic function by tissue Doppler imaging and clinical status in children with hypertrophic cardiomyopathy. Circulation 2004;109:1756-62.

29. Geske JB, Sorajja P, Nishimura RA, Ommen SR. Evaluation of left ventricular filling pressures by Doppler echocardiography in patients with hypertrophic cardiomyopathy: correlation with direct left atrial pressure measurement at cardiac catheterization. Circulation 2007; 116:2702-8.

30. Nagueh SF, Lakkis NM, Middleton KJ, Spencer WH $3^{\text {rd }}$, Zoghbi WA, Quiñones MA. Doppler estimation of left ventricular filling pressures in patients with hypertrophic cardiomyopathy. Circulation 1999;99:254-61.

31. Leask A. Potential therapeutic targets for cardiac fibrosis: TGFbeta, angiotensin, endothelin, CCN2, and PDGF, partners in fibroblast activation. Circ Res 2010;106:1675-80.

32. Eisenberg LM, Markwald RR. Molecular regulation of atrioventricular valvuloseptal morphogenesis. Circ Res 1995;77:1-6.

33. Broberg CS, Chugh SS, Conklin C, Sahn DJ, Jerosch-Herold M. Quantification of diffuse myocardial fibrosis and its association with myocardial dysfunction in congenital heart disease. Circ Cardiovasc Imaging 2010;3:727-34.

34. Coats AJ, Shewan LG. Statement on authorship and publishing ethics in the International Journal of Cardiology. Int J Cardiol 2011;153:239-40.

35. Kaufman BD, Auerbach S, Reddy S, et al. RAAS gene polymorphisms influence progression of pediatric hypertrophic cardiomyopathy. Hum Genet 2007;122:515-23.
36. O'Leary PW, Durongpisitkul K, Cordes TM, et al. Diastolic ventricular function in children: a Doppler echocardiographic study establishing normal values and predictors of increased ventricular end-diastolic pressure. Mayo Clin Proc 1998;73:616-28.

37. Eidem BW, McMahon CJ, Cohen RR, et al. Impact of cardiac growth on Doppler tissue imaging velocities: a study in healthy children. J Am Soc Echocardiogr 2004;17:212-21.

38. Breunis WB, Biezeveld MH, Geissler J, et al. Vascular endothelial growth factor gene haplotypes in Kawasaki disease. Arthritis Rheum 2006;54:1588-94.

39. Buraczynska M, Ksiazek P, Baranowicz-Gaszczyk I, Jozwiak L. Association of the VEGF gene polymorphism with diabetic retinopathy in type 2 diabetes patients. Nephrol Dial Transplant 2007;22:827-32.

40. Hlatky MA, Quertermous T, Boothroyd DB, et al. Polymorphisms in hypoxia inducible factor 1 and the initial clinical presentation of coronary disease. Am Heart J 2007;154:1035-42.

41. Howell WM, Ali S, Rose-Zerilli MJ, Ye S. VEGF polymorphisms and severity of atherosclerosis. J Med Genet 2005;42:485-90.

42. Pan D, Li Q, Jiang N, Liu A, Yu K. Robust joint analysis allowing for model uncertainty in two-stage genetic association studies. BMC Bioinformatics 2011;12:9.

43. Thomas DC, Casey G, Conti DV, Haile RW, Lewinger JP, Stram DO. Methodological Issues in Multistage Genome-wide Association Studies. Stat Sci 2009;24:414-29. 